\title{
pSmad 3D Localization in Zebrafish Embryos
}

\author{
Madeline $\mathrm{Ku}^{1}$, Xu Wang ${ }^{2}$, David Umulis ${ }^{2}$
}

${ }^{1}$ Indiana University School of Medicine, ${ }^{2}$ Purdue University, Purdue's Weldon School of Biomedical Engineering, ${ }^{2}$ Purdue University, Purdue's Weldon School of Biomedical Engineering,

\section{Background and Hypothesis}

During embryonic development, individual cell fate is determined by morphogen gradient surrounding a particular cell. In particular, the positional information regarding dorsalventral pole is determined by the BMP concentration gradient and the anterior-posterior positional information is determined by Nodal concentration gradient. pSmad is a downstream product of BMP signaling that plays a role in the feedback regulation of BMP. Previously, we had taken confocal images of the immunohistochemistry labeled pSmad embryos on its side view (yolk sac facing the side, where ventral and dorsal pole is visible) to understand the localization of pSmad and its role in BMP signaling. However, signals diminished in the deeper part of the embryos making it harder to distinguish signals from the background noise. In this experiment, we decided to use wavelet segmentation method for image processing that will help us pick up the signals better and to reconstruct the embryos from 3 different views. We hypothesized by adding these 2 procedures, it will give us a more accurate localization of pSmad signaling in zebrafish embryos.

\section{Experimental Design or Project Methods}

We first used immunohistochemistry to label pSmad in 38 hours post fertilization zebrafish embryos. We then used Zeiss DM800 confocal to image the immunohistochemistry labeled pSmad embryos in 3 different views (pole - yolk facing towards camera, ventral, and side). These images are processed through wavelet segmentation method using Matlab, then reconstruct the half embryo from the 3 views rotation. We will then compare the result we obtained compare to the raw files and results we obtained from previous experiments.

\section{Results}

Thus far, we had obtained 3 views on 3 different embryos and complete the wavelet segmentation processing. We had yet to merge the 3 views to reconstruct the embryos and compare to previous results.

\section{Conclusion and Potential Impact}

This study will establish a more accurate localization studying method on immunohistochemistry labeled zebrafish embryos that is not limited to pSmad signaling. It can be used to build mathematical model of BMP feedback regulation signaling. 